'Departamento de Cirugía Digestiva, Hospital Clínico Pontificia Universidad Católica, Facultad de Medicina Pontificia Universidad Católica de Chile. Santiago, Chile. ${ }^{2}$ Escuela de Medicina, Facultad de Medicina Pontificia Universidad Católica de Chile. Santiago, Chile.

Trabajo no recibió financiamiento.

Los autores declaran no tener conflictos de interés.

Recibido el 10 de abril de 2021, aceptado el 12 de octubre de 2021.

Correspondencia a: Cristóbal Azócar Bizama Calle Diagonal Paraguay 362, Departamento de Cirugía Digestiva, Santiago. cazocar1@uc.cl

\section{Recidiva intestinal única de melanoma cutáneo maligno: reporte de un caso}

\author{
FRANCISCO NAVARRO', MARÍA INÉS GAETE², \\ CHRISTOPHE RIQUOIR ${ }^{2}$, CRISTÓBAL AZÓCAR ${ }^{1}$, \\ FERNANDO PIMENTEL ${ }^{1}$
}

\section{Intestinal relapse of a cutaneous melanoma. Report of one case}

\begin{abstract}
Digestive tract primary melanoma is uncommon. However, metastatic lesions are more frequent and occur mainly in the small intestine. We report a 69-year-old male patient who consulted for a hyperpigmented skin lesion on the left thigh associated with multiple subcutaneous nodules. The biopsy was compatible with melanoma and PET/CT was positive for metastases in nodules and in an inguinal lymph node. Radiotherapy and chemotherapy with pembrolizumab were performed with good response, associated with posterior resection of the inguinal lymph node and melanocytic lesions. At three years offollow-up, a new hypermetabolic focus in the proximal jejunum was found in a control PET/CT. An endoscopic biopsy confirmed that it was a recurrence of the melanoma. Laparoscopic resection with primary anastomosis was performed with good clinical evolution. The definitive biopsy showed a melanoma metastasis with two of three lymph nodes positive for metastasis and a non-mutated BRAF gene. In conclusion, a single intestinal recurrence of melanoma is rare and requires an active search, since it can be resected using minimally invasive techniques.
\end{abstract}

(Rev Med Chile 2021; 149: 1236-1240)

Key words: Intestinal Neoplasms; Melanoma; Neoplasm Metastasis.

\section{E} 1 melanoma es un tumor maligno de origen principalmente cutáneo, cuya incidencia global es cercana a 2,8 por 100.000 habitantes, con una mortalidad de 0,6 por 100.000 , sin diferencias globales por género ${ }^{1}$. Se presenta por lo general después de los 65 años, sin embargo, puede afectar a cualquier grupo etario ${ }^{2}$. El melanoma gastrointestinal primario es raro y se da preferentemente a nivel anorrectal ${ }^{3}$, mientras que las lesiones metastásicas aparecen con mayor frecuencia en intestino delgado ${ }^{4}$. Habitualmente son asintomáticos, diagnosticándose como hallazgo en el seguimiento posterior con imágenes de un melanoma cutáneo tratado. El objetivo del presente artículo es presentar un caso infrecuente de recidiva intestinal única de melanoma cutáneo maligno en el yeyuno y realizar una revisión de la literatura disponible.

\section{Caso clínico}

Paciente de género masculino de 69 años consulta por hallazgo de una lesión cutánea verrucosa e hiperpigmentada en muslo izquierdo de dos años de evolución con posterior aparición de múltiples nódulos subcutáneos satelitales asociados a adenopatía inguinal ipsilateral de $3 \mathrm{~cm}$. Se realiza biopsia de lesión, la que resulta compatible con melanoma nodular S-100 (+), Melan A (+), SOX-10 (+) 
CD99 (+) focal en membrana, Sinaptofisina (-), Desmina (-), NRAS mutado en codón 61, BRAF no mutado. Tomografía por emisión de positrones con tomografía computada (PET/CT) demuestra una adenopatía inguinal izquierda, nódulos subcutáneos en muslo ipsilateral y en pierna derecha, todos con actividad hipermetabólica sugerente de metástasis. Se complementa el estudio con una resonancia magnética (RM) de cerebro que descarta lesiones metastásicas a este nivel. Biopsia excisional pierna derecha compatible con metástasis de melanoma conocido. Estadio cTxN3M1. Se decide iniciar tratamiento con intención curativa con radioterapia y 4 ciclos de Pembrolizumab, con buena respuesta en PET/CT de control. En agosto de 2018 se realiza vaciamiento inguinal izquierdo y resección de lesiones en muslo ipsi- lateral, evidenciando compromiso de linfonodos (4/10) e infiltración nodular hipodérmica por melanoma. Posteriormente, el paciente completa 35 ciclos de Pembrolizumab a finales de Marzo de 2020. En Enero de 2021, PET/CT de control evidencia un nuevo foco hipermetabólico a nivel de yeyuno proximal a $10-15 \mathrm{~cm}$ del ángulo de Treitz, asociado a una adenopatía mesentérica adyacente (Figura 1), sin presencia de otras lesiones. En endoscopía digestiva alta (EDA) se evidencia lesión proliferativa, ulcerada, con zonas hiperpigmentadas a nivel de yeyuno proximal de $3,3 \mathrm{~cm}$ con biopsia positiva para metástasis de melanoma (Figura 2). Se realiza una resección oncológica laparoscópica de la lesión yeyunal con márgenes de 5 $\mathrm{cm}$, más resección del mesenterio correspondiente $\mathrm{y}$ anastomosis primaria (Figuras 3 y 4 ). El paciente

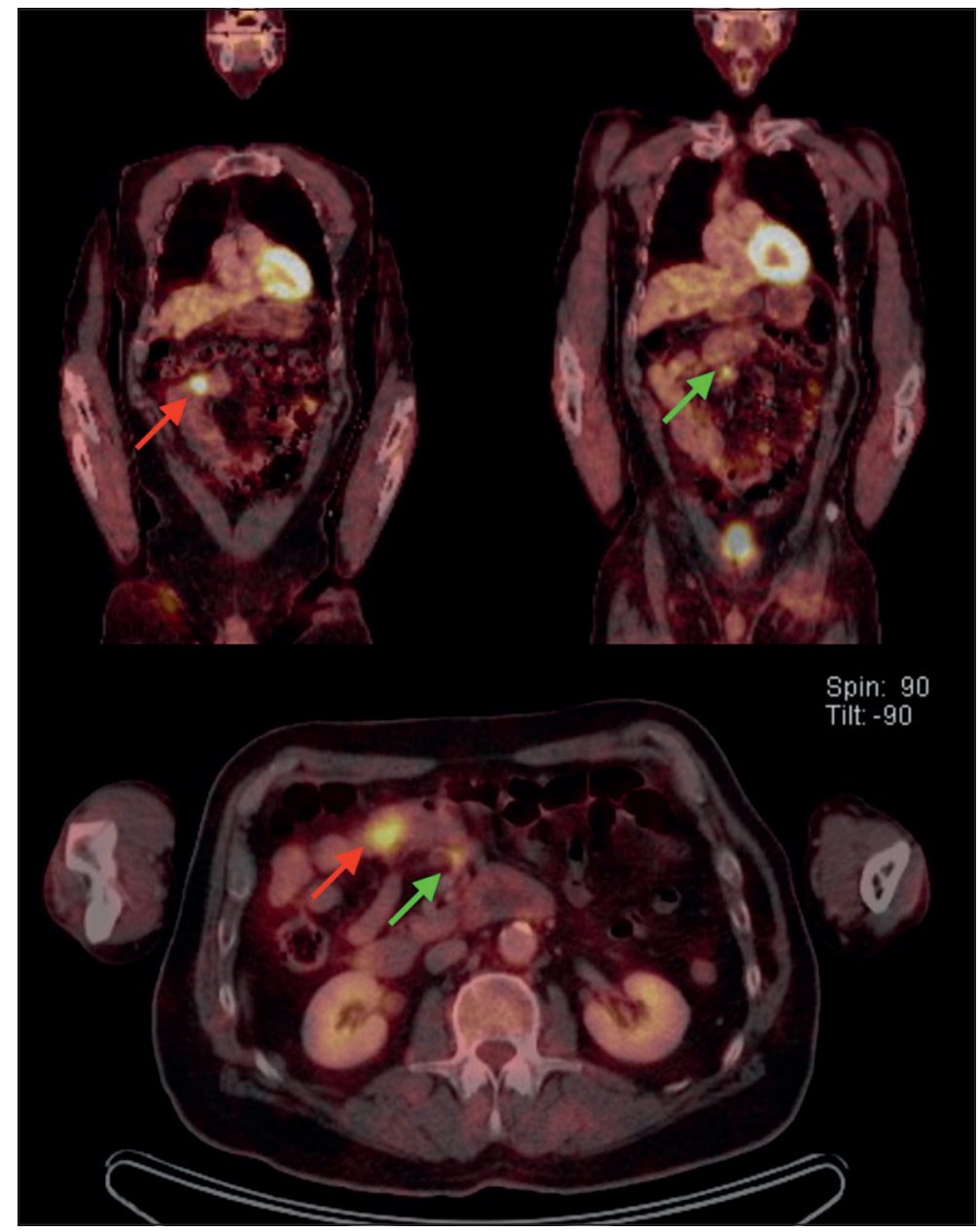

Figura 1. $P E T / C T$ : foco hipermetabólico en asas de yeyuno proximal a $10-15 \mathrm{~cm}$ del ángulo de Treitz con SUV máximo de 8,8 (flecha roja) y adenopatía mesentérica hipermetabólica adyacente con SUV máximo de 4,8 (flecha verde), compatibles con metástasis. 


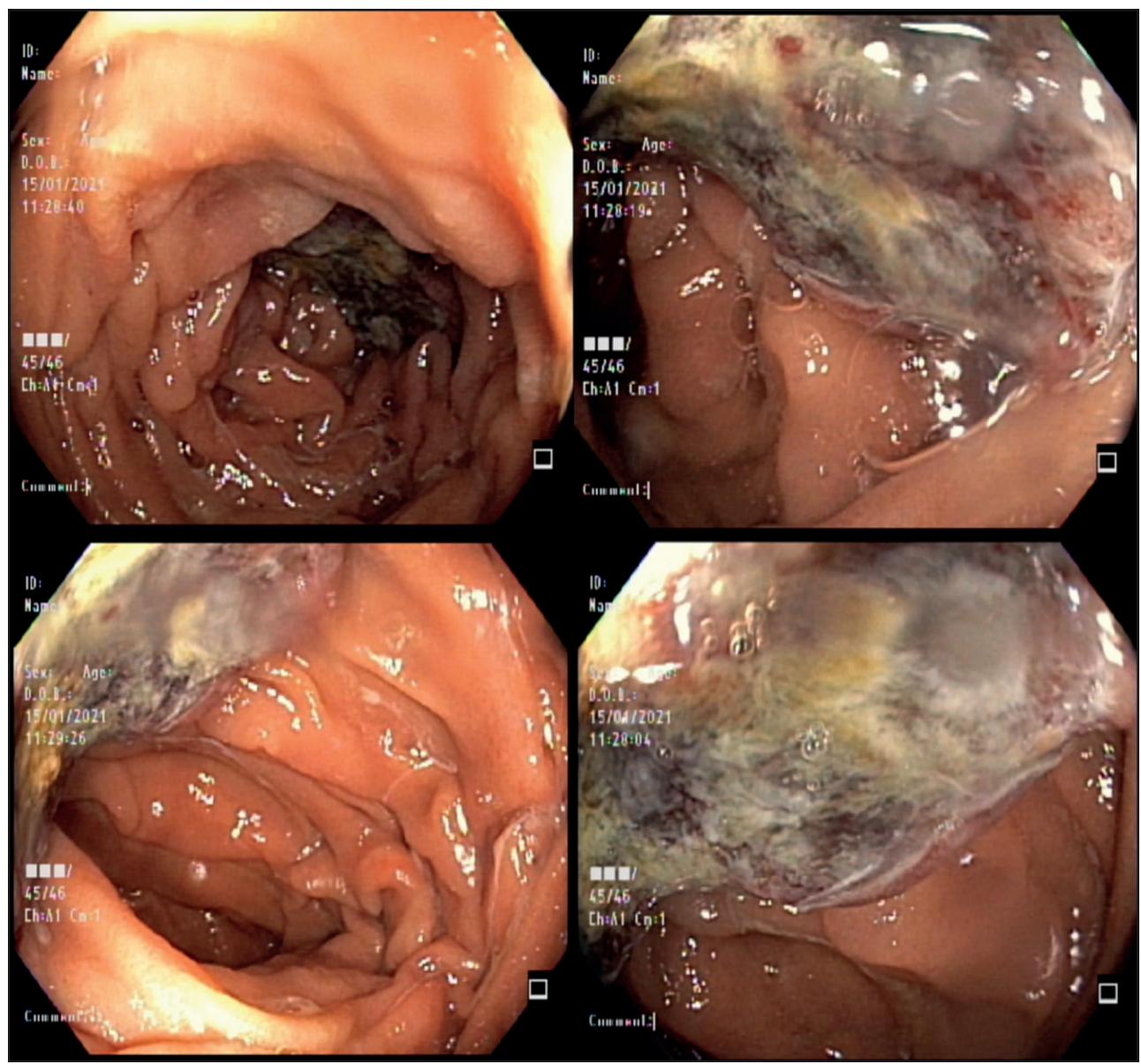

Figura 2. Endoscopía digestiva alta: lesión proliferativa hiperpigmentada de $20 \mathrm{~mm}$, ulcerada, que compromete el $40 \%$ de la circunferencia en yeyuno proximal.

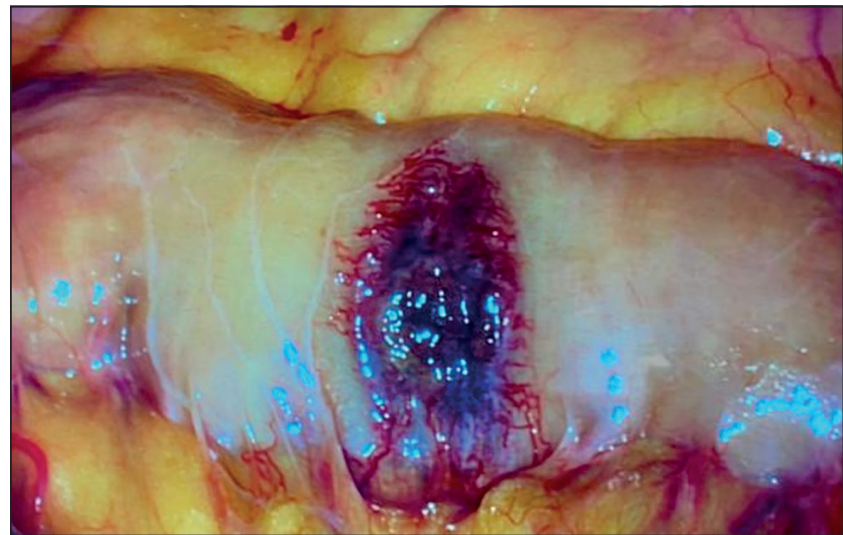

Figura 3. Laparoscopía exploradora: lesión hiperpigmentada e hipervascularizada a nivel del yeyuno proximal sin compromiso de planos adyacentes.

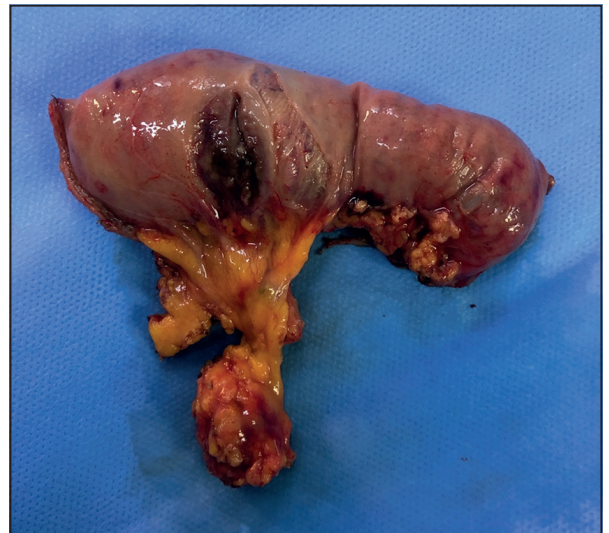

Figura 4. Pieza operatoria: lesión hiperpigmentada en segmento de yeyuno proximal con márgenes de $5 \mathrm{~cm}$ y meso con linfonodos correspondientes. 
evoluciona de forma favorable, siendo dado de alta al tercer día postoperatorio. La biopsia definitiva evidencia metástasis de melanoma, transmural, ulcerada, de $3,3 \mathrm{~cm}$ en segmento de yeyuno con 2/3 linfonodos positivos para metástasis y BRAF no mutado. El consentimiento informado para la publicación de este caso clínico fue firmado presencialmente por el paciente y adjuntado a los documentos enviados para estos fines.

\section{Discusión}

El melanoma es el tumor primario que con mayor frecuencia genera compromiso metastásico intestinal, en alrededor de 50-70\% de todos los $\operatorname{casos}^{5,6}$.

El melanoma primario de intestino delgado es extremadamente infrecuente. Una de las hipótesis para su desarrollo, propuesta por Amar en 1992, incluye la migración de células melanoblásticas de la cresta neural hacia el íleon distal a través del conducto onfalomesentérico ${ }^{5}$. Sin embargo, otros autores han cuestionado la existencia del melanoma primario intestinal argumentando que, en presencia de alguna lesión en dicha zona, ésta probablemente sea metastásica de un primario oculto o resecado previamente ${ }^{14}$. Por otro lado, resulta difícil diferenciar entre melanoma primario o metastásico considerando sólo sus características histopatológicas ${ }^{4}$.

Los melanomas se diseminan en primer lugar a pulmón y en segundo lugar a tracto gastrointestinal ${ }^{7}$. Es así, como en hasta un $4 \%$ de los pacientes diagnosticados con melanoma es posible detectar compromiso gastrointestinal, ya sea en la endoscopía o en el estudio de imágenes ${ }^{8}$. Los sitios más frecuentes son el intestino delgado (51$71 \%)$, estómago $(27 \%)$ y colon $(22 \%)^{9}$. A su vez, se ha descrito que cerca de $50 \%$ de los pacientes que fallecen por melanoma metastásico, tiene compromiso intestinal ${ }^{10}$.

Dentro de los subtipos de melanoma, el de extensión superficial es el más frecuente y también el que con mayor probabilidad genera metástasis intestinales ${ }^{11}$. El tiempo de desarrollo desde el diagnóstico del melanoma primario varía de entre los 2 meses hasta los 15 años, siendo la mayoría sólo detectados en estudios de autopsia ${ }^{12}$.

El hecho de que las metástasis sean en primer lugar a nivel de intestino delgado podría estar ex- plicado por la expresión del receptor CCR9 en las células tumorales de melanoma, que poseen una alta afinidad por la citoquina CCL25, altamente expresada en este epitelio ${ }^{13}$. Es así como a nivel intestinal, el sitio más frecuente corresponde al íleon, con más de $80 \%$ de los casos y presencia de hasta $75 \%$ de metástasis a linfonodos mesentéricos adyacentes $^{6}$.

La mayoría de los melanomas intestinales primarios o metastásicos se presentan en forma asintomática o con sintomatología inespecífica como dolor abdominal, hemorragia digestiva o baja de peso ${ }^{15}$, pudiendo llegar en algunos casos hasta la intususcepción o perforación intestinal ${ }^{11}$. En el presente caso el paciente fue totalmente asintomático y la lesión fue un hallazgo en el seguimiento imagenológico.

El uso de tomografía computada (TC) para el diagnóstico de metástasis intestinales de melanoma alcanza una sensibilidad cercana a $66 \%{ }^{16}$, pero carece de especificidad. El PET-TC es más sensible y permite al mismo tiempo la detección del tumor primario. La endoscopía permite detectar algunas lesiones, sin embargo, es inadecuada para la exploración completa del tracto intestinal y tampoco permite detectar lesiones extraluminales o que no sean transmurales, alcanzando una sensibilidad de $65-70 \%{ }^{17}$. La cápsula endoscópica es una opción en estos casos, aunque dado su alto costo y la imposibilidad de obtener muestras histológicas lo convierten en una alternativa de menor uso.

Respecto al tratamiento, la European Society for Medical Oncology (ESMO) recomienda la resección quirúrgica asociada a terapia sistémica adyuvante para los pacientes con etapa III y IV resecables $^{18}$, logrando reducir la siembra hematógena ${ }^{19} \mathrm{y}$ alcanzando una sobrevida global mediana de 5,5 años.

La terapia sistémica en estos casos consiste en el uso de bloqueadores del receptor PD-1 (nivolumab, pembrolizumab) como primera línea. En el subgrupo de pacientes con BRAF mutado, los inhibidores de BRAF y MEF tendrían beneficio en la modulación inmune en pacientes con melanoma avanzado ${ }^{21}$.

La sobrevida media de pacientes con metástasis viscerales no pulmonares es menor a 1 año. Sin embargo, se ha visto que la resección completa de estas lesiones logrando una cirugía oncológica R0 mejora la sobrevida a 5 años hasta $38-41 \%{ }^{10}$. No obstante, algunos estudios en pacientes con 
melanomas primarios de intestino delgado no han logrado demostrar un beneficio en la sobrevida con cirugía ${ }^{22}$, lo cual podría estar explicado por la presencia de un melanoma primario oculto.

En conclusión, el melanoma es uno de los tumores que con mayor frecuencia genera metástasis intestinales, siendo el yeyuno una localización infrecuente, aún más como una recidiva intestinal única. Es fundamental la búsqueda activa de lesiones en el tiempo, dado que si son únicas se pueden resecar mediante técnicas mínimamente invasivas, logrando obtener pacientes considerados tratados. Pese a esto, la mayoría de las localizaciones secundarias son múltiples y de un mal pronóstico oncológico.

\section{Referencias}

1. International Agency for Reserch on Cancer. Melanoma of skin ASR. Glob Cancer Obs. 2019; 1-2.

2. CancerResearchUK. National Cancer Registration and Analysis Service, Public Health England. Risks and causes of melanoma. 2020; 1-6.

3. Blecker D, Abraham S, Furth EE, Kochman ML. Melanoma in The Gastrointestinal Tract. Am J Gastroenterol. 1999; 94 (12): 3427-33.

4. Lens M, Bataille V, Krivokapic Z. Melanoma of the small intestine. Lancet Oncol. 2009; 10 (5): 516-21.

5. Amar A, Jougon J, Edouard A, Laban P, Marry JP, Hillion G. Primary malignant melanoma of the small intestine. Gastroenterol Clin Biol. 1992; 16 (4): 365-7.

6. Krüger S, Noack F, Blöchle C, Feller AC. Primary malignant melanoma of the small bowel: a case report and review of the literature. Tumori. 2005; 91 (1): 73-6.

7. Patel JK, Didolkar MS, Pickren JW, Moore RH. Metastatic pattern of malignant melanoma. A study of 216 autopsy cases. Am J Surg. 1978; 135 (6): 807-10.

8. Backman H. Metastases of malignant melanoma in the gastrointestinal tract. Geriatrics. 1969; 24 (8): 112-20.

9. López RG, Santomé PM, Porto EI, Moreiras MIP, Gómez CG, Villanueva JMC, et al. Intestinal perforation due to cutaneous malignant melanoma mestastatic implants. Vol. 103, Revista espanola de enfermedades digestivas : organo oficial de la Sociedad Espanola de Patologia Digestiva. Spain; 2011. p. 386-8.
10. Ollila DW, Essner R, Wanek LA, Morton DL. Surgical resection for melanoma metastatic to the gastrointestinal tract. Arch Surg. 1996; 131 (9): 975-80.

11. Patel K, Ward ST, Packer T, Brown S, Marsden J, Thomson $\mathrm{M}$, et al. Malignant melanoma of the gastro-intestinal tract: A case series. Int J Surg. 2014;12(5):523-7.

12. Berger AC, Buell JF, Venzon D, Baker AR, Libutti SK. Management of symptomatic malignant melanoma of the gastrointestinal tract. Ann Surg Oncol. 1999; 6 (2): 155-60.

13. Amersi FF, Terando AM, Goto Y, Scolyer RA, Thompson JF, Tran AN, et al. Activation of CCR9/CCL25 in cutaneous melanoma mediates preferential metastasis to the small intestine. Clin cancer Res an Off J Am Assoc Cancer Res. 2008; 14 (3): 638-45.

14. Elsayed AM, Albahra M, Nzeako UC, Sobin LH. Malignant melanomas in the small intestine: a study of 103 patients. Am J Gastroenterol. 1996; 91 (5): 1001-6.

15. Wysocki WM, Komorowski AL, Darasz Z. Gastrointestinal metastases from malignant melanoma: report of a case. Surg Today. 2004; 34 (6): 542-6.

16. Liang K V, Sanderson SO, Nowakowski GS, Arora AS. Metastatic malignant melanoma of the gastrointestinal tract. Mayo Clin Proc. 2006; 81 (4): 511-6.

17. Ricaniadis N, Konstadoulakis MM, Walsh D, Karakousis CP. Gastrointestinal metastases from malignant melanoma. Surg Oncol. 1995; 4 (2): 105-10.

18. Michielin O, van Akkooi ACJ, Ascierto PA, Dummer R, Keilholz U. Cutaneous melanoma: ESMO Clinical Practice Guidelines for diagnosis, treatment and follow-up $\dagger$. Ann Oncol Off J Eur Soc Med Oncol. 2019; 30 (12): 1884-901.

19. Roth JA, Silverstein MJ, Morton DL. Metastatic potential of metastases. Surgery. 1976; 79 (6): 669-73.

20. Hsueh EC, Gupta RK, Yee R, Leopoldo ZC, Qi K, Morton DL. Does endogenous immune response determine the outcome of surgical therapy for metastatic melanoma? Ann Surg Oncol. 2000; 7 (3): 232-8.

21. Ribas A, Algazi A, Ascierto PA, Butler MO, Chandra S, Gordon M, et al. PD-L1 blockade in combination with inhibition of MAPK oncogenic signaling in patients with advanced melanoma. Nat Commun. 2020; 11 (1): 6262.

22. Cheung MC, Perez EA, Molina MA, Jin X, Gutierrez JC, Franceschi D, et al. Defining the role of surgery for primary gastrointestinal tract melanoma. J Gastrointest Surg Off J Soc Surg Aliment Tract. 2008; 12 (4): 731-8. 